\title{
Therapeutic targeting of hypoxia and hypoxia inducible factor 1 with acriflavine in experimental esophageal adenocarcinoma
}

\author{
Christian Lankford ${ }^{1}$, Md Sazzad Hassan ${ }^{1}$ and Urs von Holzen ${ }^{1,2}$ \\ ${ }^{1}$ Indiana University School of Medicine-South Bend, South Bend, IN; ${ }^{2}$ Goshen Center for \\ Cancer Care, Goshen, IN
}

Background: Hypoxia or insufficient tissue oxygenation contributes to cancer aggressiveness and poor clinical prognosis. Typically, cancer cell lines are used in two-dimensional (2D) cultures for screening of anticancer agents, and they do not represent the hypoxic tumor microenvironment. In this study, we established a novel three dimensional (3D) spheroid coculture model of esophageal adenocarcinoma (EAC). We then observed treatment response of chemo and hypoxia-targeting therapies in 2D, 3D and in vivo models of experimental EAC. Methods: EAC 3D spheroids were generated from co-culturing human EAC and fibroblast cell lines. NanoCulture ${ }^{\circledR}$ plates and dishes were used for 3D spheroid cultures. Hypoxic status was detected by adding hypoxia probe LOX-1 and fluorescent microscopy. Paclitaxel (PT), carboplatin (CP) and nanoparticle albumin-bound paclitaxel (NPT) were used as chemotherapeutic agents, whereas acriflavine was used as hypoxia-targeting agent. In vitro cell growth was detected by WST-1 assay, in vivo tumor growth was detected by measuring subcutaneous xenografts, apoptosis was detected by cleaved caspase 3/PARP expressions, and hypoxia-targeting was detected by HIF-1 $\alpha$ expression. Results: 3D culture was more resistant to antiproliferative effect of chemotherapeutic agents PT, CP, NPT over 2D monolayer culture. Contrary to that, hypoxiatargeting agent acriflavine showed stronger antiproliferative effects in 3D culture than in 2D culture. We observed strong expression of hypoxia inducible factor-1 $\alpha$ (HIF-1 $\alpha$ ) in 3D culture with no expression of HIF-1 $\alpha$ in 2D culture, and acriflavine treatment completely abolished the HIF-1 $\alpha$ expression in 3D culture. We also observed hypoxia inside the 3D culture spheroids, but not in cells grown in 2D culture. In addition, acriflavine showed significant in vivo antitumor efficacy both as monotherapy and in combination with NPT. Conclusion: Thus 3D cultures may be better than 2D cultures in simulating the important in vivo tumor characteristic of hypoxia, and HIF-targeting therapy acriflavine could be a novel treatment strategy for EAC. 